\title{
Hysteresis in Transport Critical-Current Measurements of Oxide Superconductors
}

\author{
L. F. Goodrich and T. C. Stauffer
}

\begin{abstract}
We have investigated hysteresis in transport critical-current $\left(I_{c}\right)$ measurements of Ag-matrix $\left(\mathrm{Bi}, \mathrm{Pb}_{2} \mathrm{Sr}_{2}-\right.$ $\mathrm{Ca}_{2} \mathrm{Cu}_{3} \mathrm{O}_{10-x}(\mathrm{Bi}-2223)$ and $\mathrm{AgMg}$-matrix $\mathrm{Bi}_{2} \mathrm{Sr}_{2} \mathrm{CaCu}_{2} \mathrm{O}_{8+\mathrm{x}}$ (Bi2212) tapes. $I_{c}$ hysteresis causes measurements with field, angle, and temperature sweeps to be multi-valued. Which value is correct is addressed in the context that the proper sequence of measurement conditions reflects the application conditions. Hysteresis in angle-sweep and temperature-sweep data is related to field-sweep hysteresis.
\end{abstract}

Index Terms - Angle, critical current, high temperature superconductors, hysteresis, variable temperature.

\section{EXPERIMENTAL PROCEDURE}

$\mathrm{T}$ HE effect of hysteresis on the measured transport critical current $\left(I_{\mathrm{c}}\right)$ of oxide high-temperature superconductors (HTS) is a very important consideration for every measurement that involves more than one sweep of magnetic field, changes in field angle, or changes in temperature at a given field. For a tape specimen, the angle, $\theta$, refers to the angle between the magnetic field-strength vector $H$ and the surface normal vector of the tape (see Fig. 1). Many papers [1]-[7] have reported $I_{c}$ hysteresis observed in transport $I_{0}$ measurements of HTS. The most common observation of hysteresis is that the measured $I_{\mathrm{c}}$ as a function of $H$ is different when measured with increasing and decreasing field. Thus, $I_{\mathrm{c}}(H)$ is a multi-valued function, as shown in Fig. 2. $I_{\mathrm{c}}$ hysteresis can be reset to an initial virgin state by heating the superconductor above its critical temperature $T_{\mathrm{c}}$.

The multi-valued nature of $I_{\mathrm{c}}$ hysteresis leads to the question, Which values are "correct"? The correct value is determined by the sequence of conditions the conductor experiences in the intended application. In a magnet, the conductor is cooled to some temperature in zero field. Then the field is increased to some maximum value, and its angle with respect to any portion of the conductor remains nearly constant. Of course the angle will be different for different portions of the magnet. Any enhanced $I_{\mathrm{c}}$ caused by the portion of the conductor being exposed to a previous higher field, a higher temperature, or other field angles is not relevant. Thus, the initial virgin values are correct since they are obtained after zero-field cooling from a temperature above $T_{\mathrm{c}}$ and settling of the angle and temperature. In this paper, the terms "virgin" or "correct" identify data taken after this sequence of conditions. This paper focuses on (a) the

Manuscript received September 18, 2000. This work was supported in part by intramural funding from the NIST Advanced Technology Program.

Both authors are with the National Institute of Standards and Technology (NIST), Boulder, CO 80305 USA (telephone: 303-497-3143, e-mail: goodrich@boulder.nist.gov).

Publication of NIST, not subject to copyright

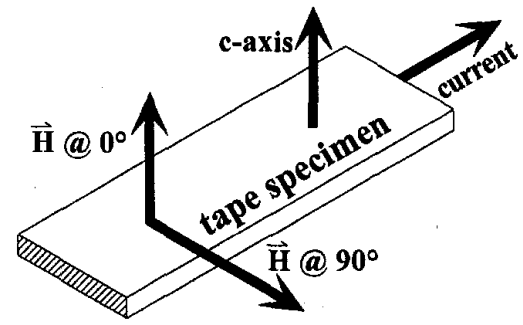

Fig. 1. Illustration of the definition of the magnetic field angle with respect to the tape-specimen geometry and current.

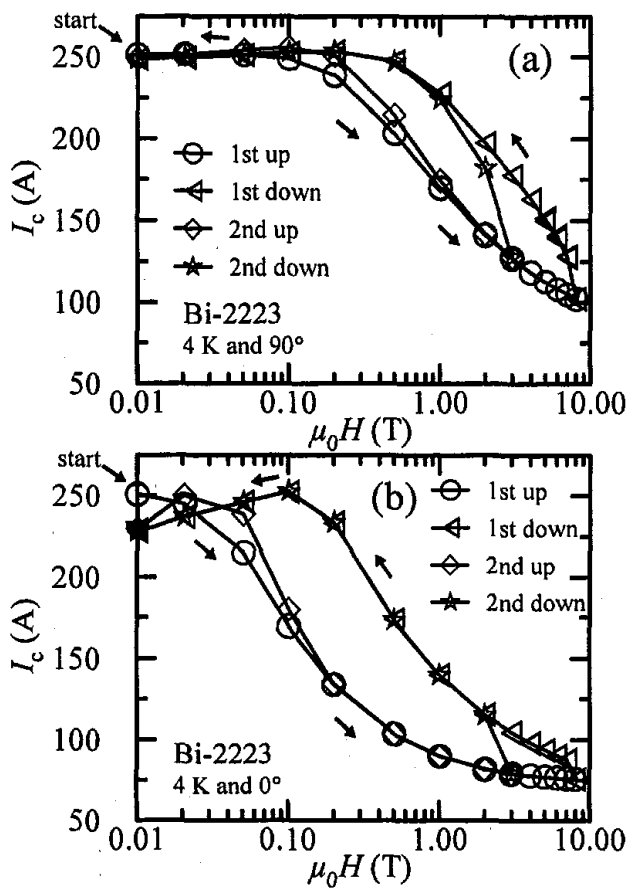

Fig. 2. $I_{\mathrm{c}}$ versus field for the $\mathrm{Bi}-2223$ specimen for various field-sweep directions, temperatures, and angles: (a) $4 \mathrm{~K}$ and $90^{\circ}$, (b) $4 \mathrm{~K}$ and $0^{\circ}$

observed $I_{\mathrm{c}}$ hysteresis, (b) what is the correct value of $I_{\mathrm{c}}$, and (c) what more expedient sequence of measurement conditions gives values that are closest to correct.

Two commercially produced multifilamentary HTS tape specimens were studied: Ag-matrix $(\mathrm{Bi}, \mathrm{Pb})_{2} \mathrm{Sr}_{2} \mathrm{Ca}_{2} \mathrm{Cu}_{3} \mathrm{O}_{10-x}$ (Bi-2223) and AgMg-matrix $\mathrm{Bi}_{2} \mathrm{Sr}_{2} \mathrm{CaCu}_{2} \mathrm{O}_{8+x}$ (Bi-2212). Measurements were made as a function of field $(0 \mathrm{~T}$ to $8 \mathrm{~T})$, angle $\left(-90^{\circ}\right.$ to $\left.90^{\circ}\right)$, and temperature $(4 \mathrm{~K}$ to $80 \mathrm{~K})$. For the Bi-2223 specimen, significant $I_{\mathrm{c}}$ hysteresis was observed in field, angle, and temperature sweeps. Much less hysteresis was observed in measurement on the $\mathrm{Bi}-2212$ specimen 
under all conditions. The source of this hysteresis and the difference between $\mathrm{Bi}-2223$ and $\mathrm{Bi}-2212$ is thought to be weaker intergrain coupling and a larger effective field at the grain boundaries in $\mathrm{Bi}-2223$ that is (a) enhanced with increasing field by the diamagnetically excluded flux from the superconductive grains, and (b) lowered with decreasing field by the trapped flux inside the grains [1], [4]. Only a small part of the measurements made on these two specimens can be shown and reported here. A more detailed paper will appear elsewhere [8].

The cryostat used in this study is a research device and a detailed description is beyond the scope of this paper. The cryostat is inserted into the $52 \mathrm{~mm}$ diameter radial-access (vertical) port of an $8 \mathrm{~T}$ split-pair magnet with a $95 \mathrm{~mm}$ diameter. (horizontal) bore. The HTS specimens were mounted on a stainless steel (AISI type 316) mandrel using a glass-filled epoxy and were soldered to copper current contacts. The distance between the current contacts was about $10 \mathrm{~cm}$, and that between the voltage taps was $5 \mathrm{~cm}$. The criterion used for all of the $I_{\mathrm{c}}$ data presented here was 0.1 $\mu \mathrm{V} / \mathrm{cm}$. The Bi-2223 specimen had a $I_{\mathrm{c}}$ of $251 \mathrm{~A}\left(J_{\mathrm{e}}=272\right.$ $\mathrm{A} / \mathrm{mm}^{2}$ ) at $0 \mathrm{~T}$ and $4 \mathrm{~K}$, with an $n$-value of 54 . The $\mathrm{Bi}-2212$ specimen had a $I_{\mathrm{c}}$ of $137 \mathrm{~A}\left(J_{\mathrm{e}}=220 \mathrm{~A} / \mathrm{mm}^{2}\right)$ at $0 \mathrm{~T}$ and $4 \mathrm{~K}$. The low-field $\mathrm{n}$-value for the $\mathrm{Bi}-2212$ specimen was 5 to 6 for temperatures of $65 \mathrm{~K}$ and below. The estimated expanded uncertainty (a two-standard-deviation estimate) of the $I_{c}$ measurement is $\pm 2 \% \pm 0.2 \mathrm{~A}$. The estimated expanded uncertainty of the angle measurement is $\pm 2^{\circ}$.

A separate heater is used to rapidly warm the center part of the specimen, not the entire apparatus, so that the specimen can be efficiently reset to the virgin state. This procedure is referred to as "flash heating" and is used to greatly decrease the length of time necessary for cooling. With this flash heater, the Bi- 2223 specimen could be heated from $4 \mathrm{~K}$ to near its $T_{\mathrm{c}}$ and cooled back to $4 \mathrm{~K}$ in less than $15 \mathrm{~min}$. Each of the specimens was cycled to near or above its $T_{c}$ more than 60 times. Some of these cycles were a gradual warming overnight, but most were done with the flash heater. The "start" arrow on Figs. 2, 5, 6, and 7 indicates the first measurement after the specimen was flash heated or cooled from a temperature higher than $120 \mathrm{~K}$.

\section{RESULTS AND DISCUSSION}

\section{A. Hysteresis with Magnetic Field Sweeps}

Fig. $2 \mathrm{a}$ is a semi-logarithmic plot of $I_{c}$ versus field for Bi2223 tape at $4 \mathrm{~K}$ and $90^{\circ}$. The measurements in zero applied field (only in self-field) were assigned an arbitrary value of $0.01 \mathrm{~T}$ to allow them to be plotted on the logarithmic scale. A start arrow points to the first value in zero field. The up and down curve segments form the two branches of the hysteresis loop that are determined by the sweep direction of the field. The points at the extremes of the sweep are plotted with multiple symbols to close the loops. These data show that $I_{c}$ at a given field ( $2 \mathrm{~T}$ ) can be about $40 \%$ higher when it is measured with monotonically decreasing fields (from a higher field) compared to monotonically increasing fields.

Hysteresis is also observed at $0^{\circ}$, as shown in Fig. $2 \mathrm{~b}$ at $4 \mathrm{~K}$. The specimen was flash heated before the initial point indicated by the start arrow. The $I_{\mathrm{c}}$ measured at zero field after a field sweep is lower than the initial $I_{\mathrm{c}}$ measured at zero field by about $9 \%$. The largest difference between the sweep direction occurs at about $0.2 \mathrm{~T}$ and is about $74 \%$.

Another way to show the field sweep hysteresis is to plot $I_{\mathrm{c}}$ measured with decreasing field divided by $I_{c}$ measured with increasing field versus field. Fig. $3 a$ and $3 b$ show these data at $90^{\circ}$ and $0^{\circ}$, respectively. The size of the hysteresis decreases with increasing temperature. For the higher fields and temperatures there is little or no hysteresis.

Field sweep hysteresis on the $\mathrm{Bi}-2212$ specimen is shown in Fig. $4 \mathrm{a}$ and $4 \mathrm{~b}$ at $90^{\circ}$ and $0^{\circ}$, respectively. At $90^{\circ}$, the largest difference in measured $I_{\mathrm{c}}$ occurs at about $3 \mathrm{~T}$ and is about $6 \%$. At $0^{\circ}$, the largest difference in measured $I_{\mathrm{c}}$ occurs between $0.2 \mathrm{~T}$ and $0.5 \mathrm{~T}$ and is about $7 \%$. The difference between zero field values is about $3 \%$ at $4 \mathrm{~K}$. The anomalous ratios at the higher fields and temperatures are due to slight differences in the small $I_{\mathrm{c}}$ values.

\section{B. Hysteresis with Angle Sweeps}

Following the above, the correct angle measurements are those obtained on the virgin field sweep with the specimen held at a fixed angle for the entire curve. These will be referred to as fixed-angle field-sweep data. Acquiring the angle dependence by angle sweeps at a given field is expedient and common. A matrix of angle sweeps were acquired in constant applied fields of $0.2 \mathrm{~T}, 1 \mathrm{~T}$, and $5 \mathrm{~T}$ and temperatures of $4 \mathrm{~K}, 20 \mathrm{~K}$, and $35 \mathrm{~K}$ for both specimens.

The hysteresis observed on the $\mathrm{Bi}-2223$ specimen with an angle sweep at $4 \mathrm{~K}$ and $5 \mathrm{~T}$ is shown in Fig. 5. The starting point is the one indicated at $90^{\circ}$ and was obtained with a fixed-angle field sweep, thus it is a virgin point. The $y$-axis is the $I_{c}$ normalized by the value at this starting point. Other
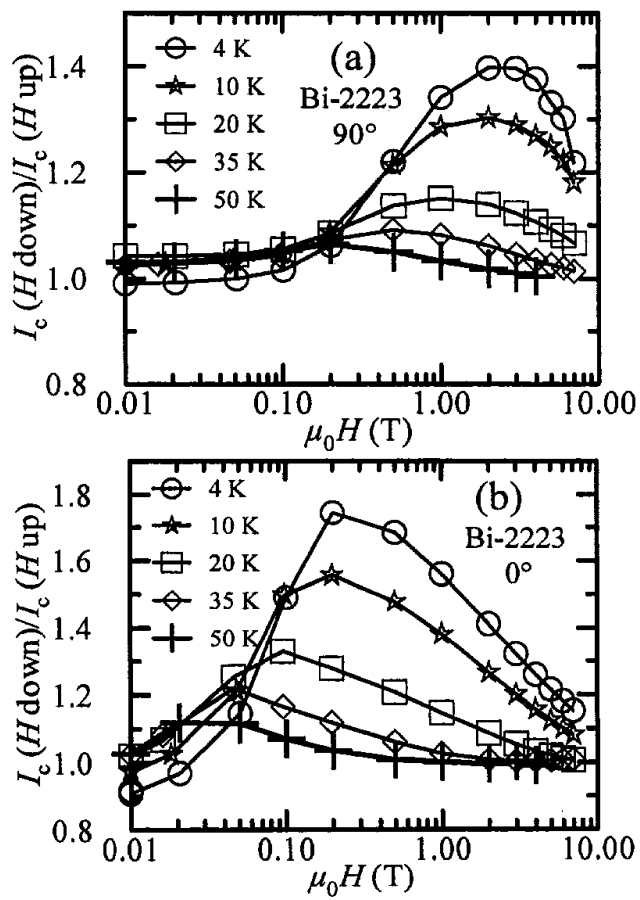

Fig. 3. $I_{c}(H$ down $) / I_{c}(H$ up) versus field for the $\mathrm{Bi}-2223$ specimen for various temperatures and angles: (a) $90^{\circ}$, (b) $0^{\circ}$. 

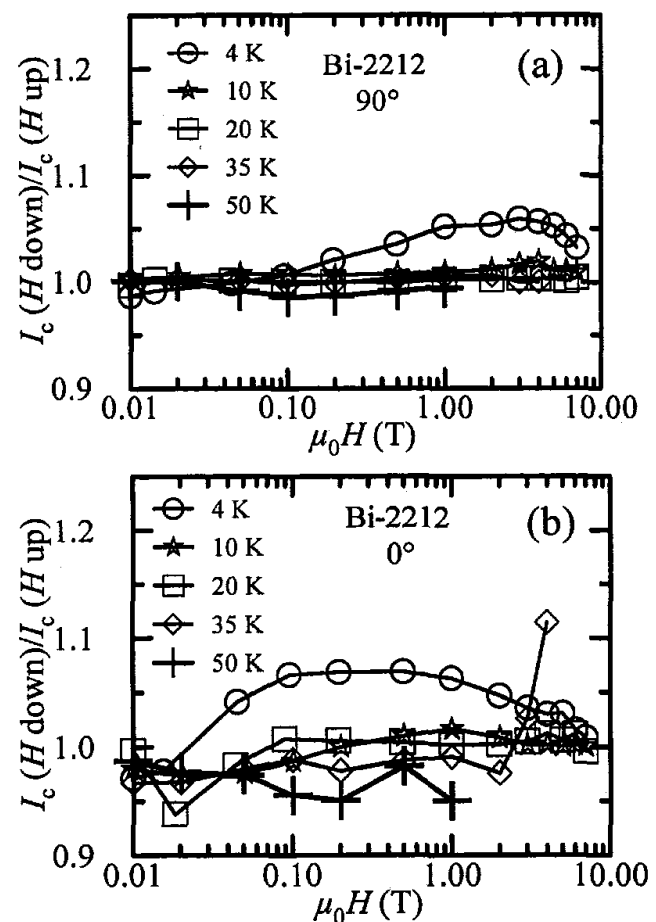

Fig. 4. $I_{\mathrm{c}}(H$ down $) / I_{\mathrm{c}}(H$ up) versus field for the Bi-2212 specimen for various temperatures and angles: (a) $90^{\circ}$, (b) $0^{\circ}$.

fixed-angle field-sweep points are also shown with the virgin $(\nabla)$ symbol. After the field angle has been swept and returned to $90^{\circ}$, the measured $I_{\mathrm{c}}$ was about $17 \%$ higher. Other than the first virgin point, the angle sweeps result in fairly reproducible hysteresis loops. For the angle-sweep data, the two branches of the hysteresis loops are determined by whether the angle is being swept towards or away from $0^{\circ}$. The measured $I_{c}$ can be $32 \%$ higher at a given field angle depending on the angle-sweep direction. The minimum $I_{\mathrm{c}}$ as a function of angle with sweep towards $0^{\circ}$ occurs at about $30^{\circ}$, and this value is nearly the same as the virgin value at $0^{\circ}$. The apparent local peak near $0^{\circ}$ is just an artifact of $I_{\mathrm{c}}$ hysteresis. The angle-sweep $I_{c}$ at $0^{\circ}$ is about $5 \%$ higher than the virgin value. The lower branches (sweep towards $0^{\circ}$ ) of the anglesweep curve yield results that are closer to the correct fixedangle data than the upper branches. A more general rule would be that the angle-sweep results are more correct when swept from higher $I_{\mathrm{c}}$ to lower $I_{\mathrm{c}}$.

Similar angle-sweep hysteresis was observed at $0.2 \mathrm{~T}$ and $1 \mathrm{~T}$ at $4 \mathrm{~K}$, and systematically less hysteresis was observed at higher temperatures and all fields. In all cases, the lower branches (sweep towards $0^{\circ}$ ) of the angle-sweep curve yield results that are closer to the virgin data than the upper branches. Also, the extra features (local maximum near $0^{\circ}$ and extra bump when approaching $90^{\circ}$ ) decrease as the amount of hysteresis decreases.

Murakami et al. [6] showed a correlation between the hysteresis observed in field sweeps and that observed in angle sweeps at a given field. Following [6], another way to plot these angle-sweep data is shown in Fig. 6. The $x$-axis of Fig. 6 is the normal component of the field $\left(\mu_{0} H \cos \theta\right)$. This

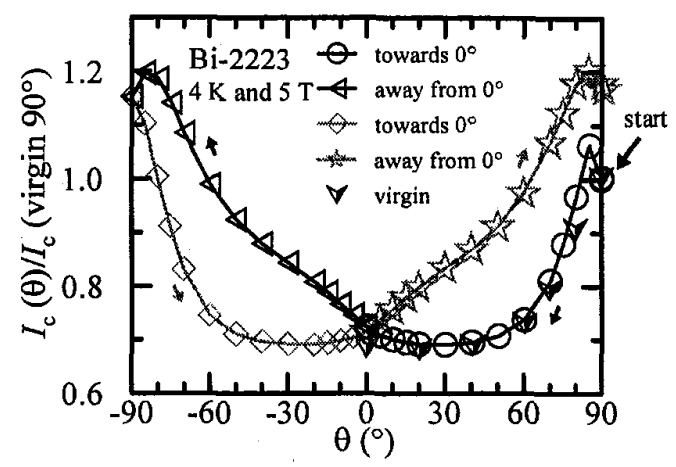

Fig. 5. $I_{\mathrm{c}} / I_{\mathrm{e}}\left(\right.$ virgin $\left.90^{\circ}\right)$ versus angle for the Bi-2223 specimen at $4 \mathrm{~K}$ and $5 \mathrm{~T}$.

folds the $+90^{\circ}$ and $-90^{\circ}$ data together and a single hysteresis loop is formed. The dashed hysteresis loop in Fig. 6 is the major loop of a field sweep from $0 \mathrm{~T}$ to $8 \mathrm{~T}$ at $0^{\circ}$ (from Fig. $2 \mathrm{~b}$ ). Most of the angle-sweep loop measured at $5 \mathrm{~T}$ is approximated by the field-sweep loop measured at $0^{\circ}$, and there is a correlation between the amount of hysteresis observed in the angle-sweep loop and that observed in the field-sweep loop. This correlation suggests that the component of the field along $0^{\circ}$ dominates the measured $I_{\mathrm{c}}$. This forms a good approximation except at the lower fields. Similar or better agreement was demonstrated for the matrix of temperatures and fields.

Much less angle-sweep hysteresis was observed on the Bi2212 specimen. There is only noticeable hysteresis in the low field data at $4 \mathrm{~K}$. The higher field and higher temperature data have very little hysteresis. In all cases, the lower branches (sweep towards $0^{\circ}$ ) of the angle-sweep curve yield results that are closer to the virgin data than the upper branches. In all cases, a $0^{\circ}$ field-sweep to an appropriate maximum field provides a good approximation to the hysteresis observed in the angle-sweep curves at a given field. But, the $0^{\circ}$ field-sweep curves slightly underestimated the observed angle-sweep hysteresis at all fields and temperatures for the $\mathrm{Bi}-2212$ specimen.

\section{Hysteresis with Temperature Sweeps}

A matrix of temperature sweeps was acquired in constant fields of $0.2 \mathrm{~T}, 1 \mathrm{~T}$, and $5 \mathrm{~T}$ and angles of $0^{\circ}$ and $90^{\circ}$ for both specimens. Figure 7 shows temperature-sweep data taken with fixed field $(1 \mathrm{~T})$ and angle $\left(0^{\circ}\right)$. Virgin values were used to normalize these data. The first-up temperature sweep yields results very close to the virgin values. This is significant only at $20 \mathrm{~K}$ and $35 \mathrm{~K}$, since the initial data at $4 \mathrm{~K}$ amounted to a repeat of the virgin conditions. This again is consistent with the general rule that more correct data are obtained by sweeping from higher $I_{c}$ to lower $I_{c}$. The temperature-sweep hysteresis is observed as the temperature is then decreased from $35 \mathrm{~K}$. The amount of temperaturesweep hysteresis scales with that observed during the field sweeps at each corresponding temperature and angle. The dashed line in Fig. 7 shows the hysteresis observed during field sweeps, which was extracted from Fig. $3 \mathrm{~b}$ and forms an envelope for the temperature sweep hysteresis. The correlation between field-sweep and temperature-sweep hysteresis suggests that sweeping to higher temperatures 


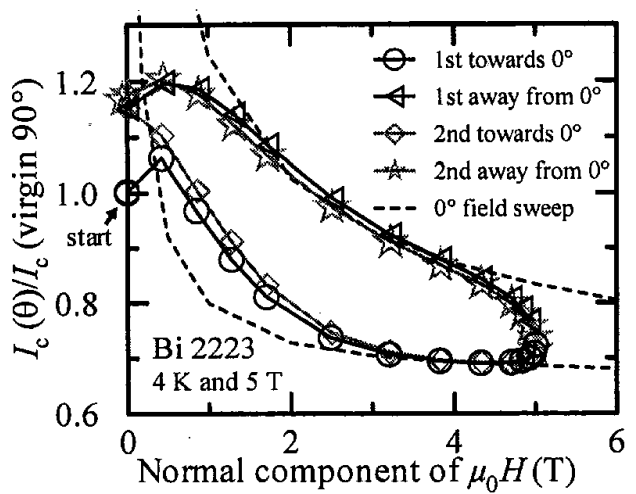

Fig. 6. $I_{\mathrm{c}} / I_{\mathrm{c}}\left(\right.$ virgin $\left.90^{\circ}\right)$ versus the normal component of the field for the Bi2223 specimen at $4 \mathrm{~K}$ and $5 \mathrm{~T}$ for the various angle sweeps. The dash curves show the $I_{\mathrm{c}}$ versus field curves at $0^{\circ}$ taken from Fig. $2 \mathrm{~b}$.

(lower $I_{\mathrm{c}}$ ) is similar to sweeping to higher fields (lower $I_{\mathrm{c}}$ ). For the field-cooled points, the specimen was flash heated and then cooled while the field was held constant. In the field-cooled case, there can be hysteresis at $35 \mathrm{~K}$. In the entire matrix, the field-cooled hysteresis was about the same or somewhat larger than observed with a temperature sweep down from $35 \mathrm{~K}$. The field-sweep envelopes also provided a good approximation for the temperature sweep hysteresis of the $\mathrm{Bi}-2212$ specimen.

The character of the hysteresis during temperature sweeps is somewhat different from that observed with field and angle sweeps. This is likely due to the fact that the field magnitude and angle do not change during these temperature sweeps. As a result of this, once the sweep departs from the more correct branch, it never gets back to the more correct branch by ramping just the temperature up and down. This is shown in Fig. 7 where the "second $T$ up" and "first $T$ down" values are nearly the same at $20 \mathrm{~K}$ (also in "field cooled, then $T$ up").

\section{CONCLUSIONS}

$I_{\mathrm{c}}$ hysteresis was studied on two commercially produced multifilamentary HTS tape specimens: Bi-2223 and Bi-2212. $I_{\mathrm{c}}$ hysteresis was observed to cause measured values to be as much as $74 \%$ higher or $9 \%$ lower than the correct value. Which $I_{\mathrm{c}}$ value is correct depends on the sequence of conditions in the application. Most applications cannot easily take advantage of the enhanced $I_{\mathrm{c}}$ observed during specimen testing due to the nature of this hysteresis. The $I_{\mathrm{c}}$ hysteresis in Bi-2223 conductors is much larger than in Bi-2212 conductors. In both cases, the size of the hysteresis effect decreases with increasing temperature.

If $I_{\mathrm{c}}$ hysteresis is observed when the field is ramped up and down, then it will exist when the field angle is swept or the temperature is swept. The observed field-sweep hysteresis can be used to estimate the hysteresis observed in both angle and temperature sweeps. Some of the features observed in angle-sweep data are artifacts of $I_{\mathrm{c}}$ hysteresis.

In general, for field, angle, and temperature sweeps, the sweep data are more correct if the sweep starts where the $I_{\mathrm{c}}$ is the highest and goes to where it is the lowest. This can be used to reduce the hysteresis effect and allow for approximate characterization to be made in a more expedient way.

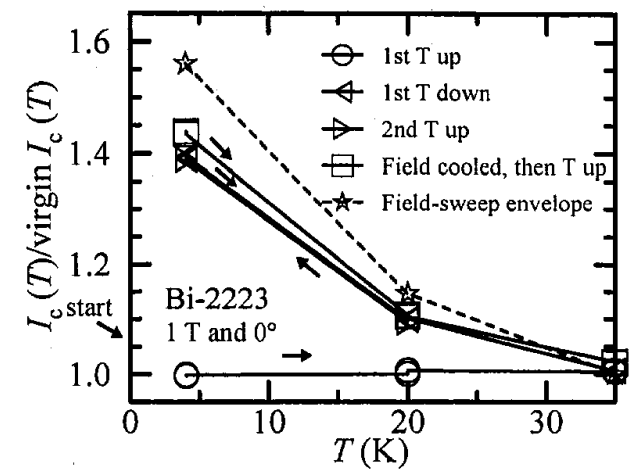

Fig. 7. $I_{\mathrm{c}}(T) /$ virgin $I_{\mathrm{c}}(T)$ versus temperature for the Bi-2223 specimen at $1 \mathrm{~T}$ and $0^{\circ}$ for various sweep directions. The dash curve shows the field-sweep hysteresis taken from Fig. 3b.

If significant $I_{\mathrm{c}}$ hysteresis exists, the correct sequence of testing conditions appropriate for most HTS applications consists of magnetic field sweeps with the specimen at a fixed angle and a fixed temperature and with the specimen first heated to near its $T_{\mathrm{c}}$ and then cooled in zero field between magnet field sweeps at different angles or temperatures.

The temperature sweep hysteresis is somewhat different from field and angle sweep hysteresis. Without a change in the applied field magnitude or angle, the temperature sweep hysteresis retains the enhanced $I_{\mathrm{c}}$, which suggest that the flux remains in the higher pinned regions during subsequent temperature sweeps.

\section{ACKNOWLEDGMENT}

We acknowledge years of support by the late S. L. Bray (NIST). We thank Ken Marken (Oxford Superconducting Technology) for providing one of the specimens. Certain materials are identified in this paper to foster understanding. Such identification does not imply recommendation or endorsement by the NIST, nor does it imply that the materials identified are necessarily the best available for the purpose.

\section{REFERENCES}

[1] J.E. Evetts and B.A. Glowacki, Relation of critical current irreversibility to trapped flux and microstructure in polycrystalline $\mathrm{YBa}_{2} \mathrm{Cu}_{3} \mathrm{O}_{7}$, Cryogenics vol. 28, pp. 641-649, Oct. 1988.

[2] K. Watanbe, K. Noto, et al., Anisotropy and hysteresis of transport critical currents in high temperature $\mathrm{Ln}-\mathrm{Y}-\mathrm{Ba}-\mathrm{Cu}-\mathrm{O}$ superconductors, Cryogenics vol. 29, pp. 263-267, Mar. 1989.

[3] L.F. Goodrich and S.L. Bray, High- $T_{\mathrm{c}}$ superconductors and the critical current measurement, Cryogenics vol. 30, pp. 667-677, Aug. 1990.

[4] K.I. Kugel and A.L. Rakhmanov, Non-uniform magnetic flux distribution in high $T_{c}$ ceramics and hysteretic behaviour of critical current, Cryogenics vol. 33, pp. 281-286, Mar. 1993.

[5] B.A. Glowacki, J. Jackiewicz, and J.E. Evetts, Angular reversibility and irreversibility of critical current in polycrystalline $\mathrm{YBa}_{2} \mathrm{Cu}_{3} \mathrm{O}_{7}$ wires, Cryogenics vol. 33, pp. 86-90, Jan. 1993.

[6] Y. Murakami, K. Itoh, M. Yuyama, and H. Wada, Field-angle dependence of critical current in Ag-sheathed $\mathrm{Bi}-2212$ and $\mathrm{Bi}-2223$ tapes, Adv. Cryo. Eng. vol. 42, pp. 529-534, 1996.

[7] L.F. Goodrich, II-4: Critical-current measurement methods for oxide superconductor tapes and wires. Part 1: Transport current method, Cryogenics, submitted for publication.

[8] L.F. Goodrich and T.C Stauffer, Magnetic hysteresis in transport critical-current measurements of oxide superconductors, J. Res. Natl. Inst. Stand. Technol., submitted for publication. 